\title{
CONTEMPORANEOUS BROADBAND OBSERVATIONS OF THREE HIGH-REDSHIFT BL LAC OBJECTS
}
M. AckermanN ${ }^{1}$, M. Ajello ${ }^{2}$, H. An ${ }^{3}$, L. Baldini ${ }^{3,4}$, G. Barbiellini ${ }^{5,6}$, D. Bastieri ${ }^{7,8}$, R. Bellazzini ${ }^{9}$, E. Bissaldi ${ }^{10}$, R. D. Blandford ${ }^{3}$, R. Bonino ${ }^{11,12}$, J. Bregeon ${ }^{13}$, R. J. Britto ${ }^{14}$, P. Bruel ${ }^{15}$, R. Buehler ${ }^{1}$, G. A. Caliandro ${ }^{3,16}$, R. A. Cameron ${ }^{3}$, M. Caragiulo ${ }^{10,17}$, P. A. Caraveo ${ }^{18}$, E. Cavazzuti $^{19}$, C. Cecchi $^{20,21}$, E. Charles ${ }^{3}$, A. Chekhtman ${ }^{22}$, G. Chiaro ${ }^{8}$, S. Ciprini ${ }^{19,20}$, J. Cohen-Tanugi ${ }^{13}$, F. Costanza ${ }^{10}$, S. Cutini ${ }^{19,20,23}$, F. D'Ammando ${ }^{24,25}$, A. De Angelis ${ }^{26}$, F. De Palma ${ }^{10,27}$, R. Desiante ${ }^{11,28}$, M. Di Mauro ${ }^{3}$, L. Di Venere ${ }^{10,17}$, A. Domínguez ${ }^{2}$, P. S. Drell ${ }^{3}$, C. Favuzzi ${ }^{10,17}$, S. J. Fegan ${ }^{15}$, E. C. Ferrara ${ }^{29}$, J. Finke ${ }^{30}$, P. Fusco ${ }^{10,17}$, F. Gargano ${ }^{10}$, D. Gasparrini ${ }^{19,20}$, N. Giglietto ${ }^{10,17}$, F. Giordano ${ }^{10,17}$, M. Giroletti ${ }^{24}$, D. Green ${ }^{29,31}$, I. A. Grenier ${ }^{32}$, S. Guiriec ${ }^{29,48}$, D. Horan ${ }^{15}$, G. Jóhannesson ${ }^{33}$,

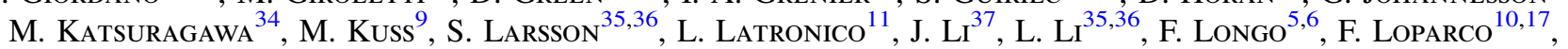
M. N. Lovellette $^{30}$, P. Lubrano ${ }^{20,21}$, J. Magill ${ }^{31}$, S. Maldera ${ }^{11}$, A. Manfreda ${ }^{9}$, M. Mayer ${ }^{1}$, M. N. Mazziotta ${ }^{10}$, P. F. Michelson ${ }^{3}$, N. Mirabal ${ }^{29,48}$, W. Mitthumsiri ${ }^{38}$, T. Mizuno ${ }^{39}$, M. E. Monzani ${ }^{3}$, A. Morselli ${ }^{40}$, I. V. Moskalenko ${ }^{3}$, M. Negro ${ }^{11,12}$, E. Nuss ${ }^{13}$, T. Ohsugi ${ }^{39}$, C. OKada ${ }^{41}$, E. Orlando ${ }^{3}$, D. Paneque ${ }^{3,42}$, M. Pesce-Rollins ${ }^{3,9}$, F. Piron $^{13}$,

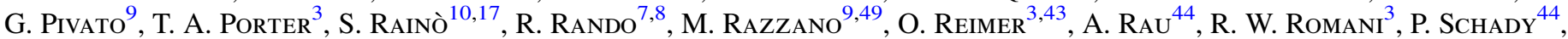
C. SGrò ${ }^{9}$, D. Simone ${ }^{10}$, E. J. Siskind ${ }^{45}$, F. Spada ${ }^{9}$, G. Spandre ${ }^{9}$, P. Spinelli ${ }^{10,17}$, D. Stern ${ }^{46}$, H. Takahashi ${ }^{41}$, J. B. ThaYer ${ }^{3}$, D. F. Torres ${ }^{37,47}$, G. Tosti ${ }^{20,21}$, E. Troja ${ }^{31,32}$, G. Vianello ${ }^{3}$, K. S. Wood ${ }^{30}$, AND M. Wood ${ }^{3}$ ${ }^{1}$ Deutsches Elektronen Synchrotron DESY, D-12738 Zeuthen, Germany

${ }^{2}$ Department of Physics and Astronomy, Clemson University, Kinard Lab of Physics, Clemson, SC 29634-0978, USA

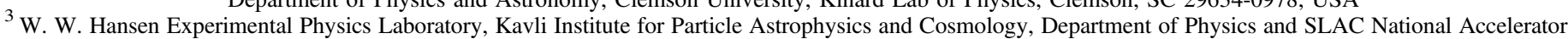
Laboratory, Stanford University, Stanford, CA 94305, USA; hjans@stanford.edu

${ }^{4}$ Università di Pisa and Istituto Nazionale di Fisica Nucleare, Sezione di Pisa I-56127 Pisa, Italy

${ }^{5}$ Istituto Nazionale di Fisica Nucleare, Sezione di Trieste, I-34127 Trieste, Italy

${ }^{6}$ Dipartimento di Fisica, Università di Trieste, I-34127 Trieste, Italy

${ }^{7}$ Istituto Nazionale di Fisica Nucleare, Sezione di Padova, I-35131 Padova, Italy

${ }^{8}$ Dipartimento di Fisica e Astronomia "G. Galilei," Università di Padova, I-35131 Padova, Italy

${ }_{9}^{9}$ Istituto Nazionale di Fisica Nucleare, Sezione di Pisa, I-56127 Pisa, Italy

${ }^{10}$ Istituto Nazionale di Fisica Nucleare, Sezione di Bari, I-70126 Bari, Italy

${ }^{11}$ Istituto Nazionale di Fisica Nucleare, Sezione di Torino, I-10125 Torino, Italy

${ }_{12}$ Dipartimento di Fisica Generale "Amadeo Avogadro," Università degli Studi di Torino, I-10125 Torino, Italy

${ }^{13}$ Laboratoire Univers et Particules de Montpellier, Université Montpellier, CNRS/IN2P3, Montpellier, France

${ }^{14}$ Department of Physics, University of Johannesburg, P.O. Box 524, Auckland Park 2006, South Africa

${ }^{15}$ Laboratoire Leprince-Ringuet, École polytechnique, CNRS/IN2P3, Palaiseau, France

${ }^{16}$ Consorzio Interuniversitario per la Fisica Spaziale (CIFS), I-10133 Torino, Italy

${ }^{17}$ Dipartimento di Fisica “M. Merlin"dell'Università e del Politecnico di Bari, I-70126 Bari, Italy

18 INAF-Istituto di Astrofisica Spaziale e Fisica Cosmica, I-20133 Milano, Italy

${ }^{19}$ Agenzia Spaziale Italiana (ASI) Science Data Center, I-00133 Roma, Italy

${ }^{20}$ Istituto Nazionale di Fisica Nucleare, Sezione di Perugia, I-06123 Perugia, Italy

21 Dipartimento di Fisica, Università degli Studi di Perugia, I-06123 Perugia, Italy

22 College of Science, George Mason University, Fairfax, VA 22030, resident at Naval Research Laboratory, Washington, DC 20375, USA

23 INAF Osservatorio Astronomico di Roma, I-00040 Monte Porzio Catone (Roma), Italy

${ }^{24}$ INAF Istituto di Radioastronomia, I-40129 Bologna, Italy

25 Dipartimento di Astronomia, Università di Bologna, I-40127 Bologna, Italy

${ }^{26}$ Dipartimento di Fisica, Università di Udine and Istituto Nazionale di Fisica Nucleare, Sezione di Trieste, Gruppo Collegato di Udine, I-33100 Udine

${ }^{27}$ Università Telematica Pegaso, Piazza Trieste e Trento, 48, I-80132 Napoli, Italy ${ }^{28}$ Università di Udine, I-33100 Udine, Italy

${ }^{29}$ NASA Goddard Space Flight Center, Greenbelt, MD 20771, USA

30 Space Science Division, Naval Research Laboratory, Washington, DC 20375-5352, USA

${ }^{31}$ Department of Physics and Department of Astronomy, University of Maryland, College Park, MD 20742, USA

${ }^{32}$ Laboratoire AIM, CEA-IRFU/CNRS/Université Paris Diderot, Service d'Astrophysique, CEA Saclay, F-91191 Gif sur Yvette, France 33 Science Institute, University of Iceland, IS-107 Reykjavik, Iceland

${ }^{34}$ Institute of Space and Astronautical Science, Japan Aerospace Exploration Agency, 3-1-1 Yoshinodai, Chuo-ku, Sagamihara, Kanagawa 252-5210, Japan

${ }^{3}$ Department of Physics, KTH Royal Institute of Technology, AlbaNova, SE-106 91 Stockholm, Sweden

The Oskar Klein Centre for Cosmoparticle Physics, AlbaNova, SE-106 91 Stockholm, Sweden

${ }^{37}$ Institute of Space Sciences (IEEC-CSIC), Campus UAB, E-08193 Barcelona, Spain
Department of Physics, Faculty of Science, Mahidol University, Bangkok 10400, Thailand

${ }^{39}$ Hiroshima Astrophysical Science Center, Hiroshima University, Higashi-Hiroshima, Hiroshima 739-8526, Japan

${ }^{40}$ Istituto Nazionale di Fisica Nucleare, Sezione di Roma "Tor Vergata," I-00133 Roma, Italy

41 Department of Physical Sciences, Hiroshima University, Higashi-Hiroshima, Hiroshima 739-8526, Japan

42 Max-Planck-Institut für Physik, D-80805 München, Germany

${ }^{43}$ Institut für Astro- und Teilchenphysik and Institut für Theoretische Physik, Leopold-Franzens-Universität Innsbruck, A-6020 Innsbruck, Austria

${ }^{44}$ Max-Planck Institut für extraterrestrische Physik, D-85748 Garching, Germany

${ }^{4}$ NYCB Real-Time Computing Inc., Lattingtown, NY 11560-1025, USA

46 Jet Propulsion Laboratory, Pasadena, CA 91109, USA

${ }^{47}$ Institució Catalana de Recerca i Estudis Avançats (ICREA), Barcelona, Spain

Received 2015 November 30; accepted 2016 February 14; published 2016 March 21

\footnotetext{
48 NASA Postdoctoral Program Fellow, USA.

49 Funded by contract FIRB-2012-RBFR12PM1F from the Italian Ministry of

Education, University and Research (MIUR).
} 


\section{ABSTRACT}

We have collected broadband spectral energy distributions (SEDs) of three BL Lac objects 3FGL J0022.1-1855 $(z=0.689)$, 3FGL J0630.9-2406 $(z \gtrsim 1.239)$, and 3FGL J0811.2-7529 $(z=0.774)$, detected by Fermi with relatively flat gigaelectronvolt spectra. By observing simultaneously in the near-infrared to hard X-ray band, we can well characterize the high end of the synchrotron component of the SED. Thus, fitting the SEDs to synchroCompton models of the dominant emission from the relativistic jet, we can constrain the underlying particle properties and predict the shape of the gigaelectronvolt Compton component. Standard extragalactic background light (EBL) models explain the high-energy absorption well, with poorer fits for high-ultraviolet models. The fits show clear evidence for EBL absorption in the Fermi spectrum of our highest-redshift source 3FGL J0630.9 -2406. While synchrotron self-Compton models adequately describe the SEDs, the situation may be complicated by possible external Compton components. For 3FGL J0811.2-7529, we also discover a nearby serendipitous source in the X-ray data, which is almost certainly another lower synchrotron peak frequency $\left(\nu_{\mathrm{pk}}^{\mathrm{sy}}\right)$ BL Lac, that may contribute flux in the Fermi band. Since our sources are unusual high-luminosity, moderate $\nu_{\mathrm{pk}}^{\mathrm{sy}}$ BL Lacs, we compare these quantities and the Compton dominance, the ratio of peak inverse Compton to peak synchrotron luminosities $\left(L_{\mathrm{pk}}^{\mathrm{IC}} / L_{\mathrm{pk}}^{\mathrm{sy}}\right)$, with those of the full Fermi BL Lac population.

Key words: BL Lacertae objects: general - BL Lacertae objects: individual (3FGL J0022.1-1855, 3FGL J0630.9 -2406, 3FGL J0811.2-7529) - galaxies: active - radiation mechanisms: non-thermal

\section{INTRODUCTION}

Blazars, active galactic nuclei (AGNs) with strong nonthermal emission from an aligned relativistic jet (Blandford \& Rees 1978; Urry \& Padovani 1995), are the most luminous persistent objects in the universe. These sources emit photons across the whole electromagnetic spectrum from the radio to gamma-ray bands. Their spectral energy distributions (SEDs) are well characterized with a double-hump structure, where the low-energy hump, peaking in the IR/optical/UV/X-ray band, is thought to be produced by synchrotron emission of the jet electrons. Their high-energy peak in the gamma-ray band is produced by synchrotron self-Compton (SSC) and external Compton (EC) scattering, or possibly by hadronic processes (e.g., Mannheim \& Biermann 1992; Boettcher et al. 1997; Ghisellini et al. 2010).

Blazars are heuristically classified into flat-spectrum radio quasars (FSRQs) and BL Lacertae objects (BL Lacs). The former show broad optical emission lines associated with clouds surrounding or in the accretion disk. The latter lack such lines and have a jet continuum strong enough to obscure spectral features of the host galaxy (Marcha et al. 1996; Landt et al. 2004). Padovani \& Giommi (1995) further divided BL Lacs based on the synchrotron peak frequency $\left(\nu_{\mathrm{pk}}^{\mathrm{sy}}\right)$ into low synchrotron peak (LSP, $\nu_{\mathrm{pk}}^{\mathrm{sy}}<10^{14} \mathrm{~Hz}$ ), intermediate peak (ISP, $10^{14} \mathrm{~Hz}<\nu_{\mathrm{pk}}^{\mathrm{sy}}<10^{15} \mathrm{~Hz}$ ), and high peak (HSP, $\left.10^{15} \mathrm{~Hz}<\nu_{\mathrm{pk}}^{\mathrm{sy}}\right)$ subclasses. FSRQs are almost all classified as LSP (Abdo et al. 2010).

Fossati et al. (1998) found that $5 \mathrm{GHz}$ luminosity, the synchrotron peak luminosity $\left(L_{\mathrm{pk}}^{\mathrm{sy}}\right)$, and the gamma-ray dominance (ratio of the peak gamma-ray to peak synchrotron $\nu F_{\nu}$ luminosity) are correlated with $\nu_{\mathrm{pk}}^{\mathrm{sy}}$. They characterize this as a "blazar sequence" trend from low-peaked powerful sources (i.e., FSRQs) to high-peaked less powerful sources (HSPs). A plausible physical explanation for this sequence is provided by Ghisellini et al. (1998); more luminous sources tend to have stronger disk accretion, and the external photons from the broad-line region (BLR) or the disk in these sources provide additional seeds for Compton upscattering, which cools the jet electrons, lowering $\nu_{\mathrm{pk}}^{\mathrm{sy}}$, while increasing the Compton luminosity. Indeed, as the typical accretion state evolves over cosmic time, this picture may provide an explanation of evolution in the FSRQ/BL Lac blazar populations (Böttcher \& Dermer 2002; Cavaliere \& D'Elia 2002). Quantitatively, this may explain the apparent "negative evolution" (increase at low redshift) observed for HSP BL Lacs (Rector et al. 2000; Beckmann et al. 2003; Ajello et al. 2014).

On the other hand, Giommi et al. (2012) used Monte Carlo simulations to argue that the $L_{\mathrm{pk}}^{\mathrm{sy}}$ and $\nu_{\mathrm{pk}}^{\mathrm{sy}}$ anticorrelation may be primarily a selection effect. Padovani et al. (2012) discuss four sources with high $\nu_{\mathrm{pk}}^{\mathrm{sy}}$ and high peak (synchrotron $+\mathrm{SSC}$ ) power as examples well away from the blazar sequence. Such sources might be FSRQs with unusually strong jet emission along the Earth line of sight, masking the underlying host components. Thus, simultaneous observations and careful SED modeling of such (generally higher redshift) BL Lac sources is interesting as they can help us understand the underlying emission-zone physics and whether it is truly different from the bulk of the blazar population. Characterization via less redshiftdependent parameters (e.g., gamma-ray dominance or Compton dominance; see Fossati et al. 1998; Finke 2013, for example) may also help clarify their place in the population. Also, comparing robust SED model fits with gamma-ray spectra of high- $z$ blazars can reveal the effect of absorption by the extragalactic background light (EBL), which provides important constraints on the evolution of cosmic star formation (e.g., Ackermann et al. 2012; H.E.S.S. Collaboration et al. 2013). BL Lacs are believed to have higher Compton dominance (CD) and less sensitivity to local soft photon fields and so are particularly useful for such studies.

Appropriate high-redshift HSP BL Lac objects are rare because they are faint, especially in the gamma-ray band, and HSPs appear to exhibit negative evolution (Ajello et al. 2014). We select three Fermi-detected (Nolan et al. 2012; Ackermann et al. 2011) sources, 3FGL J0022.1-1855 (J0022, $z=0.774)$, 3FGL J0630.9-2406 (J0630, $z>1.239$ ), and 3FGL J0811.2 -7529 (J0811, $z=0.689$ ), whose optical spectra are unusual, showing no emission lines but a set of strong low-excitation ( $\mathrm{Mg}_{\text {I }}, \mathrm{Fe}$ II, $\mathrm{Al}$ II, and so on) absorption lines on a blue, powerlaw continuum. These indicate that the AGN is viewed through the disk of an intervening absorber. In Shaw et al. (2013), this was taken to be the host galaxy; indeed for J0630 the photometry of Rau et al. (2012) supports this as the host redshift. With estimated redshifts of $0.774,>1.239$, and 0.689 (Rau et al. 2012; Shaw et al. 2013) for J0022, J0630, and 
Table 1

Summary of Observations Used in This Work

\begin{tabular}{|c|c|c|c|c|c|c|c|}
\hline Source & R.A. & Decl. & Redshift & Observatory & $\begin{array}{c}\text { Start Date } \\
\text { (MJD) }\end{array}$ & Obs. ID & $\begin{array}{c}\text { Exposure } \\
(\mathrm{ks})\end{array}$ \\
\hline \multirow{4}{*}{$\mathrm{J} 0022$} & \multirow{4}{*}{$0^{\mathrm{h}} 22^{\mathrm{m}} 09.25^{\mathrm{s}}$} & \multirow{4}{*}{$-18^{\circ} 53^{\prime} 34^{\prime \prime} 9$} & \multirow{4}{*}{0.774} & GROND & 57031.1 & $\ldots$ & $0.25 / 0.24^{\mathrm{a}}$ \\
\hline & & & & Swift & 57031.7 & 00080777001 & $1.9^{\mathrm{b}}$ \\
\hline & & & & $X M M$ & 57026.8 & 0740820501 & $15 / 9^{c}$ \\
\hline & & & & NUSTAR & 57026.7 & $60001141002-4$ & 110 \\
\hline \multirow{4}{*}{ J0630 } & \multirow{4}{*}{$6^{\mathrm{h}} 30^{\mathrm{m}} 59.515^{\mathrm{s}}$} & \multirow{4}{*}{$-24^{\circ} 06^{\prime} 46^{\prime \prime} 09$} & \multirow{4}{*}{$>1.239$} & GROND & 56949.2 & $\cdots$ & $0.25 / 0.24^{\mathrm{a}}$ \\
\hline & & & & Swift & 56948.5 & 00080776001 & $0.27^{\mathrm{b}}$ \\
\hline & & & & $X M M$ & 56948.2 & 0740820401 & $8 / 4^{c}$ \\
\hline & & & & NUSTAR & 56947.7 & 60001140002 & 67 \\
\hline \multirow{4}{*}{ J0811 } & \multirow{4}{*}{$8^{\mathrm{h}} 11^{\mathrm{m}} 03.214^{\mathrm{s}}$} & \multirow{4}{*}{$-75^{\circ} 30^{\prime} 27^{\prime \prime} 85$} & \multirow{4}{*}{0.689} & GROND & 56903.3 & $\cdots$ & $0.25 / 0.24^{\mathrm{a}}$ \\
\hline & & & & SWIFT & 56908.2 & 00091903001 & $0.39^{\mathrm{b}}$ \\
\hline & & & & $X M M$ & 56901.2 & 0740820601 & $9 / 6^{\mathrm{c}}$ \\
\hline & & & & NUSTAR & 56901.2 & 60001142002 & 113 \\
\hline
\end{tabular}

Notes.

${ }^{a}$ For $g^{\prime} r^{\prime} i^{\prime} z^{\prime} / J H K$ bands.

${ }^{\mathrm{b}}$ For the UW1 band. Exposures in the other UVOT bands may differ from this value.

${ }^{\mathrm{c}}$ For MOS1, 2/PN.

J0811, respectively, these are thus luminous high-peak sources suitable for studying the extreme of the BL Lac population. At these redshifts, we may also see the effects of EBL absorption at the high end of the Fermi band. To probe this absorption and the high end of the jet particle population most sensitive to Compton cooling, we require a particularly good characterization of the peak and high-energy cutoff (near-IR to hard X-ray) of the synchrotron component. Under classic SSC modeling, this allows us to characterize the high-energy Compton component as well, thus providing inferences about the Compton cooling at the source and EBL absorption of the gigaelectronvolt photons as they propagate to Earth.

In this paper, we present broadband SEDs of the three highredshift BL Lacs that are simultaneous across the critical $\nu>\nu_{\mathrm{pk}}^{\mathrm{sy}}$ range (Section 2). J0630 has been previously discussed as a high- $\nu_{\mathrm{pk}}^{\mathrm{sy}}$, high-power source (Padovani et al. 2012); our improved data allow more refined modeling, which is discussed in Section 3, including EBL constraints. The implications of our inferred model parameters are discussed in Section 4. We use $H_{0}=70 \mathrm{~km} \mathrm{~s}^{-1} \mathrm{Mpc}^{-1}, \Omega_{m}=0.3$, $\Omega_{\Lambda}=0.7$ (e.g., Komatsu et al. 2011), and the redshift values given in Table $1(z=1.239$ for J0630) throughout.

\section{OBSERVATIONS AND DATA REDUCTION}

BL Lac objects can be variable on all timescales from minutes to years (Aleksić et al. 2015), so coordinated broadband coverage is important for characterizing the instantaneous SED. We therefore carried out nearly contemporaneous observations of the sources using the gamma-ray burst Optical/Near-Infrared Detector (GROND) instrument at the $2.2 \mathrm{~m}$ MPG telescope at the ESO La Silla Observatory (Greiner et al. 2008), as well as the Swift (Gehrels et al. 2004), XMM-Newton (Jansen et al. 2001), and NuSTAR (Harrison et al. 2013) satellites, covering the upper range of the synchrotron component. Our sources showed relatively modest variability in the Fermi (Atwood et al. 2009) band, so we average over 6 years of Large Area Telescope (LAT) data to best characterize the mean Compton component of these relatively faint (but luminous, for BL Lacs) sources. Archival radio, optical, and near-IR observations are provided for comparison, although we do not use them in the SED fitting.

\subsection{Contemporaneous Observations: GROND, Swift, XMM-Newton, and NuSTAR}

The GROND data were reduced and analyzed with the standard tools and methods described in Krühler et al. (2008). The photometric data were obtained using FWHM-matched point-spread function (PSF) $\left(g^{\prime} r^{\prime} i^{\prime} z^{\prime}\right)$ or aperture photometry $(J H K)$. The $g^{\prime}, r^{\prime}, i^{\prime}$, and $z^{\prime}$ photometric calibration was obtained via standard star fields observed on the same nights as the target integrations. The $J, H$, and $K s$ photometry was calibrated against selected in-field 2MASS stars (Skrutskie et al. 2006).

For the Swift UVOT data, we performed aperture photometry for the six Swift filters (Poole et al. 2008) using the uvotsource tool in HEASOFT ${ }^{50}$ 6.16. We measured the photometric magnitude of the sources using an $R=5^{\prime \prime}$ aperture. Backgrounds were estimated using an $R=20^{\prime \prime}$ circle near the source.

X-ray SEDs of the sources were measured with $X M M$ Newton and NuSTAR. The sources were detected with very high significance $(>20 \sigma)$ with XMM-Newton but with relatively low significance $(\gtrsim 6 \sigma)$ with $N u S T A R$. For the $X M M$-Newton data, we processed the observation data files with epproc and emproc of the Science Analysis System (SAS) version ${ }^{51} 14.0 .0$ and then applied standard filters. The NUSTAR data were processed with the standard pipeline tool nupipeline of nustardas 1.4.1 integrated in HEASOFT 6.16. We used NuSTAR CALDB version 20140414 and applied the standard filters. ${ }^{52}$ We then extracted source events using circular regions with $R=20^{\prime \prime}$ and $R=30^{\prime \prime}$ for the XMMNewton and the NUSTAR data, respectively. Backgrounds were extracted from nearby source-free regions.

\footnotetext{
50 http://heasarc.nasa.gov/lheasoft/

51 http://xmm.esac.esa.int/sas/

52 See http://heasarc.gsfc.nasa.gov/docs/nustar/analysis/nustar_swguide. pdf for more details.
} 


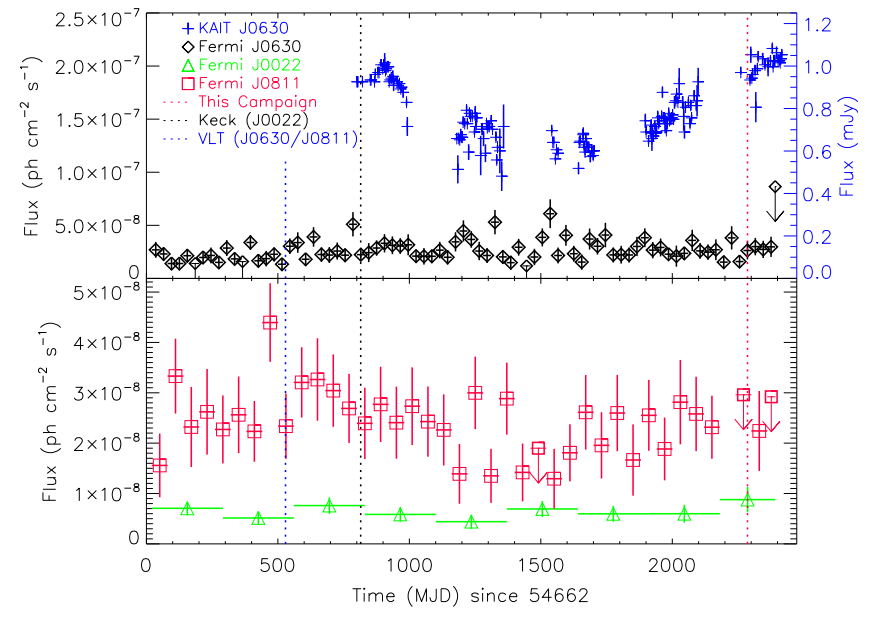

Figure 1. Optical ( $R$-band) and gamma-ray (100 MeV to $500 \mathrm{GeV}$ ) light curves. The top panel shows KAIT (right scale) and Fermi (left scale) fluxes for J0630. Our contemporaneous observation epoch and the optical spectrum epochs are marked. The lower panel shows the LAT light curves and multiwavelength epochs for J0022 and J0811. The modest LAT variability justifies the use of mission-averaged spectra.

\subsection{Gamma-ray Observations}

For the gamma-ray data, we used the Fermi observations taken between 2008 August 4 and 2015 January 31. The Pass 8 data (Atwood et al. 2013), based on a complete and improved revision of the entire LAT event-level analysis, were downloaded from the Fermi Science Support Center ${ }^{53}$, and we analyzed the data using the Fermi Science tool 10-00-04 along with the instrument response functions P8R2_SOURCE_V6. We extracted source class events in the $100 \mathrm{MeV}$ to $500 \mathrm{GeV}$ band in an $R=5^{\circ}$ region of interest (ROIs) and $<80^{\circ}$ zenith angle and $<52^{\circ}$ rocking angle cuts. These events were analyzed using the background models ( $\mathrm{gl} \mathrm{I}_{-} \mathrm{iem}$ _v06 and iso_P8R2_SOURCE_V6_V06) and all 3FGL sources within $15^{\circ}$. We first modeled fluxes on a one-month cadence to check for strong source variability using the standard Fermi likelihood analysis with gtlike (see Figure 1 and Section 3.1). No strong flares were seen, so we combined all of the LAT data, modeling the mission-averaged spectrum. In Figure 1, we mark the epochs of the contemporaneous campaign and the historical spectra. For J0630 we also have access to optical monitoring from the KAIT program (Cohen et al. 2014), shown on the top panel. Variability is clearly seen in the optical band.

\subsection{Archival Observations}

For comparison, we also collected archival data in the radioto-UV band. We assembled data from various catalogs (e.g., WISE and 2MASS for IR data) or reanalyze the archival data (e.g., VLT/Keck spectra and Swift UVOT). For the catalog data, we convert the magnitude to flux appropriately. The VLT/Keck data reduction and calibration were described in Shaw et al. (2013). The archival UVOT data are processed as described above (Section 2.1). The measurements are corrected for Galactic extinction in constructing the SED (Section 3.2). Archival measurements are used only in flux variability studies.

\footnotetext{
53 http://fermi.gsfc.nasa.gov/ssc/
}

\subsection{Discovery of a Serendipitous Source}

We discovered a serendipitous X-ray source (J0810) in the field of J0811 (Figure 2). The X-ray (XMM-Newton) position of the source is R.A. $=08^{\mathrm{h}} 10^{\mathrm{m}} 03^{\mathrm{s}}$ and decl. $=-75^{\circ} 27^{\prime} 21^{\prime \prime} \quad(\mathrm{J} 2000$, $\delta_{\text {R.A.,decl. }}=2^{\prime \prime}$ statistical only), only $6^{\prime}$ from J0811 (Figure 2 left). We find that the spectrum cannot be described with a simple absorbed power law $\left(\chi^{2} /\right.$ dof $\left.=185 / 118, p=7 \times 10^{-5}\right)$. A broken power-law model ${ }^{54}$ explains the data $\left(\chi^{2} /\right.$ dof $=116 / 116$, $p=0.47)$, and the best-fit parameters are $N_{\mathrm{H}}=1.4 \pm$ $0.3 \times 10^{21} \mathrm{~cm}^{-2}$, low-energy photon index $\Gamma_{1}=3.4 \pm 0.3$, high-energy photon index $\Gamma_{2}=1.74 \pm 0.07$, break energy $E_{\text {break }}=1.46 \pm 0.08 \mathrm{keV}$, and $3-10 \mathrm{keV}$ flux $F_{3-10 \mathrm{keV}}=$ $2.7 \pm 0.2 \times 10^{-13} \mathrm{erg} \mathrm{s}^{-1} \mathrm{~cm}^{-2}$.

Together with archival radio, optical, and Swift UV data, we construct the SED of the source (Figure 2 right). If we use the best-fit X-ray $N_{\mathrm{H}}$, the extrapolated spectrum poorly matches the optical. Instead we de-absorb using the value from the optical/ UV extinction $N_{\mathrm{H}}=6.9 \times 10^{20} \mathrm{~cm}^{-2}$. X-ray fits with absorption fixed at this value are statistically acceptable (null hypothesis probability $p=0.3$ ). The SED of this source suggests a blazar with $\nu_{\mathrm{pk}}^{\mathrm{sy}}$ in the optical range and a rise to a Compton component in the hard X-ray band. Its location in the WISE color-color diagrams (Figure 2 middle; see also D'Abrusco et al. 2012) suggests that the source should be a BL Lac. If the Compton component peaks at $>100 \mathrm{MeV}$, this source may contribute to the J0811 SED since the source is within the aperture we used for J0811. If we free the position of J0811 in the Fermi analysis, we find a maximum likelihood coincident with J0811 (magenta circle in Figure 2 left). Also, a second source at the J0810 position does not significantly increase the model test statistic (TS).

We then increased the zenith angle cut to $<100^{\circ}$ to have more events and used a small spatial bin size $(0.05)$ to see if J0810 is detected in the Fermi band. We performed a binned likelihood analysis with the new data. In this case, a gamma-ray counterpart of J0810 is detected significantly ( $\mathrm{TS}=56$ ); the model without $\mathrm{J} 0810$ is only $0.03 \%$ as probable as the one with J0810. In the $0.1-500 \mathrm{GeV}$ band, J0810 has $20 \%$ of the flux (with $40 \%$ flux uncertainty) of J0811 with a similar power-law index $\left(\Gamma_{\gamma}=1.8 \pm 0.1\right)$. These spectral parameters for $\mathrm{J} 0810$ may not be very accurate because of mixing from the brighter source, J0811. Since J0811 is brighter than J0810 in the gamma-ray band, we attribute all of the LAT flux to J0811 in the SED modeling and discuss implications of J0810 contamination on the model (see Section 3.3).

\section{DATA ANALYSIS AND RESULTS}

\subsection{Variability}

We have examined the collected data for variability because short timescales can give useful constraints on the characteristic size of the emission zone in the various wave bands. We first examined our contemporaneous data sets for short-timescale variations. For the XMM-Newton and NuSTAR data, spanning $\sim 10-100 \mathrm{ks}$, we constructed exposure-weighted light curves using various time bin sizes $(\sim 100-20,000$ s), ensuring $>20$ counts in each time bin, and calculated $\chi^{2}$ for a constant flux. The probability for constancy was always high ( $210 \%)$, implying no significant short-term variability for any of the

\footnotetext{
54 http://heasarc.gsfc.nasa.gov/docs/xanadu/xspec/manual/ XSmodelBknpower.html
} 

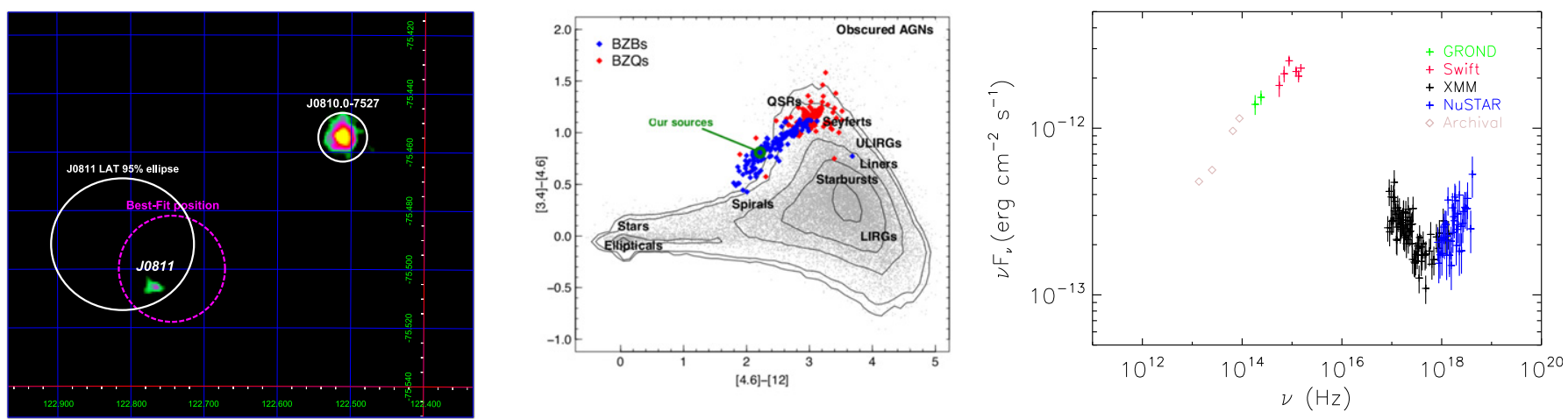

Figure 2. Left: NUSTAR image of the field containing J0811. The color scale is arbitrarily adjusted for better visibility. The Fermi/LAT 3FGL ellipse (95\%, white) and the best-fit circle (95\%, magenta) are shown, and an $R=30^{\prime \prime}$ circle is drawn around the serendipitous source (denoted as J0810.0-7527). Middle: location of the sources we are studying in the WISE [3.4]-[4.6]-[12] $\mu \mathrm{m}$ color-color diagram (figure taken from D'Abrusco et al. 2012). The four sources, including J0810, are located in the middle of the BZB (naming convention for BL Lac in the ROMA-BZCAT catalog) distribution. See D'Abrusco et al. (2012) for more detail. Right: observed SED of the serendipitous source. Note that we used $N_{\mathrm{H}}=6.9 \times 10^{20} \mathrm{~cm}^{-2}$, the optical extinction inferred value, for constructing the SED. Notice that this new source is quite hard, emitting more strongly in the NUSTAR band than in the XMM-Newton band.

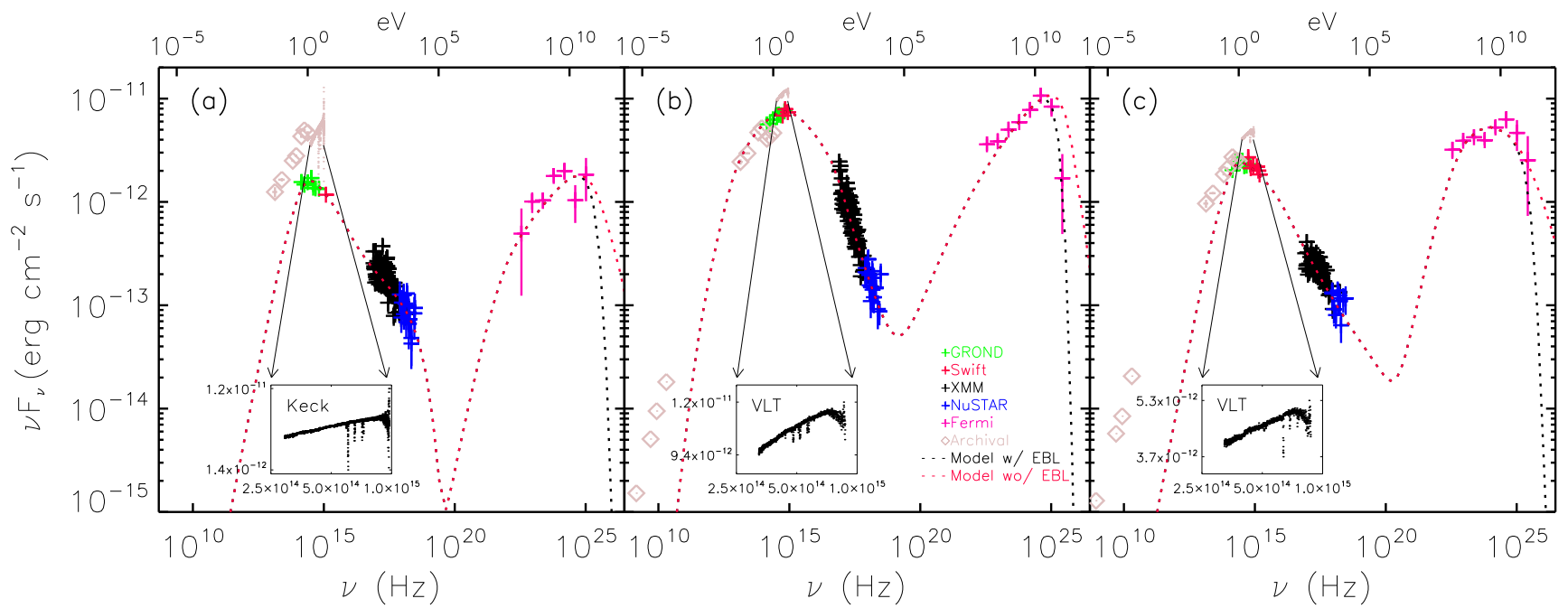

Figure 3. Observed broadband SED and best-fit models for (a) J0022, (b) J0630, and (c) J0811. Data points with error bars are taken from the contemporaneous observations (Sections 2.1 and 2.2), and diamonds are from the archival observations (Section 2.3). The dashed lines are the best-fit SSC SED models of Boettcher et al. (1997) with (black) and without (red) EBL absorption (Finke et al. 2010). Note that the archival data are not taken contemporaneously even if they are plotted in the same color and symbol. The insets plot the VLT/Keck spectra of Shaw et al. (2013), showing the lack of emission lines and the low excitation absorption complexes placing lower limits on the redshift. These observations appear to have been in a brighter, harder optical state.

three sources at this epoch. Similarly, the optical/UV data from the contemporaneous epoch did not show subday variability.

However, on longer timescales, the optical synchrotron peak flux does show substantial variability, as can be seen by comparing the contemporaneous and archival points in Figures 1 and 3. J0022, for example, varies by $\sim 6 \times$. As noted, the VLT/Keck spectra also appear to represent brighter epochs, although slit losses limit the precision of the flux calibration. In general, the brighter epochs appear to have harder near-IR to UV spectra, suggesting increased electron energy (or increased bulk Doppler factor) in flaring events. A much better characterization of J0630's optical variability is available from the KAIT Fermi AGN monitoring data (Cohen et al. 2014). ${ }^{55}$ The dominant modulation is slow on year timescales; this is of modest amplitude compared to other BL Lacs $(\sim 50 \%)$. KAIT resolves times as short as the $\sim 3$ days cadence, and we do see statistically significant $(\gtrsim \sigma \sigma)$ changes

\footnotetext{
55 http://brando.astro.berkeley.edu/kait/agn/
}

between consecutive observations. This suggests that at least some of the jet flux arises in compact $r<10^{16} \mathrm{~cm}$ structures.

We can use the LAT band to probe variability in the Compton peak emission. Since these sources are not very bright, we were able to only probe month timescales. To this end, we generated light curves by fitting source fluxes to $100 \mathrm{MeV}$ to $500 \mathrm{GeV}$ photons from a $5^{\circ} \mathrm{ROI}$ about each source using the gtlike tool for each time bin. For this we fixed the background model normalization and the background source spectral parameters at the mission-averaged values (see below), allowing only the source flux to vary with the spectral index held fixed at the values given in Table 2 . Figure 1 shows the corresponding light curves. The variability is not strong $\left(\chi^{2} /\right.$ dof values for a constant light curve of $5 / 8,92 / 72$, and $28 / 33$ for J0022, J0630, and J0811, respectively). We confirm the results of the 3FGL catalog (Acero et al. 2015); our sources are not flagged as variable in the 3FGL catalog at a $99 \%$ confidence. Finally, an examination of the light curves 
Table 2

Galactic Foreground Reddening Values and X-ray and Gamma-ray Fit Results

\begin{tabular}{lcccc}
\hline \hline Source & & $\mathrm{J} 0022$ & $\mathrm{~J} 0630$ & $\mathrm{~J} 0811$ \\
\hline$E(B-V)$ & $(\mathrm{mag})$ & 0.024 & 0.056 & 0.125 \\
$N_{\mathrm{H}, \text { Dust }}{ }^{\mathrm{a}}$ & $\left(10^{20} \mathrm{~cm}^{-2}\right)$ & 1.3 & 3.1 & 7 \\
$N_{\mathrm{H}}$ & $\left(10^{20} \mathrm{~cm}^{-2}\right)$ & $4(1)$ & $13(1)$ & $7(1)$ \\
$\Gamma_{\mathrm{X}}{ }^{\mathrm{b}}$ & $\ldots$ & $2.55(6)$ & $2.98(7)$ & $2.45(7)$ \\
$F_{\mathrm{X}}$ & $\ldots$ & $0.93(8)$ & $1.6(1)$ & $1.4(1)$ \\
$\Gamma_{\gamma}$ & $\ldots$ & $1.86(6)$ & $1.83(3)$ & $1.93(4)$ \\
$F_{\gamma}{ }^{\mathrm{c}}$ & $\ldots$ & $6.3(9)$ & $25(2)$ & $23(2)$ \\
\hline
\end{tabular}

Notes.

${ }^{\text {a }}$ Dust-extinction equivalent $N_{\mathrm{H}}$, converted with $N_{\mathrm{H}}=1.8 \times 10^{21} A(V) \mathrm{cm}^{-2}$ $\mathrm{mag}^{-1}$ and $R_{V}=3.1$ (Predehl \& Schmitt 1995).

b $3-10 \mathrm{keV}$ flux in units of $10^{-13} \mathrm{erg} \mathrm{s}^{-1} \mathrm{~cm}^{-2}$.

${ }^{c} 0.1-500 \mathrm{GeV}$ flux in units of $10^{-9}$ photons $\mathrm{s}^{-1} \mathrm{~cm}^{-2}$.

assembled by the Agenzia Spaziale Italiana science data center $^{56}$ also shows no significant variability in any source. We conclude that the three sources have been relatively quiescent for BL Lacs: this gives us confidence that the mission-averaged LAT spectrum may be usefully compared with our contemporaneous campaign fluxes for SED fitting.

\subsection{Constructing Broadband SEDs}

Next we assembled broadband SEDs for the sources using the data described in Section 2. The optical/UV magnitudes were corrected for the dust map extinction in these directions (Table 2) obtained from the NASA/IPAC extragalactic database, using the Schlafly \& Finkbeiner (2011) calibration. We show the SEDs in Figure 3. Note that Ly $\alpha$ forest absorption was visible in J0630 at frequencies above $\sim 10^{15} \mathrm{~Hz}$ in the UVOT data, as expected from its large redshift; we do not use the high-frequency UVOT data $\gtrsim 10^{15} \mathrm{~Hz}$ in the J0630 SED modeling.

The X-ray response files are produced with the standard tools in SAS and in nustardas for the XMM-Newton and NuSTAR spectra, respectively. We fit the spectra in the $0.3-79 \mathrm{keV}$ band with an absorbed power-law model in XSPEC 12.8.2 and found that the model describes the data well, having $\chi^{2} /$ dof $\lesssim 1$ for all three sources. The fact that all $\mathrm{X}$-ray spectra are well modeled by a single absorbed power law is important to the modeling below. The absorption corrections for the X-ray data were obtained from the $N_{\mathrm{H}}$ in the power-law fits. The fit results are presented in Table 2.

While the X-ray fit and extinction-map values for the absorption agree well for J0811, J0022 and especially J0630 show stronger X-ray absorption. Given the modest dust map resolution and the $\sim 50 \%$ conversion uncertainties (e.g., Gorenstein 1975; Watson 2011; Foight et al. 2015), the discrepancy for J0022 may be reconciled. However, the large value for J0630 seems difficult to accommodate, and we have no clear explanation. The Galactic HI column density ${ }^{57}$ toward J0630 is $7-12 \times 10^{20} \mathrm{~cm}^{-2}$, consistent with the X-ray-inferred value. If we assume the $\mathrm{X}$-ray value for de-extinction of the optical, we find an unnatural UV flux rise (similarly, using the optical value makes an unnatural cutoff in the low-energy Xray spectrum). Thus we can only accommodate the X-ray fit value if the optical/UV flux has an extra blue, narrow-band

\footnotetext{
56 http://www.asdc.asi.it/fermi3fgl/

57 https://heasarc.gsfc.nasa.gov/cgi-bin/Tools/w3nh/w3nh.pl
}

component. This seems unnatural. Alternatively, the dust map extinction might be correct, and the X-ray component may be spatially separated from the optical emission, experiencing extra local (host) absorption. Measurements of the J0630 VLT absorption line strengths indicated that the intervening/host galaxy supplies negligible extinction $E(B-V)<0.01$ to the optical component, which is consistent with the low effective $E(B-V)$. Acknowledging this inconsistency, we use the two values in Table 2 when constructing the SED.

For the Fermi SED, we performed a binned likelihood analysis using the same configuration as described in Section 2.2 with the 6.5 years of data. In doing so, we fit spectra for all bright sources (detected with $\gtrsim 5 \sigma$ ) in the ROI and the background amplitudes. The spectral parameters for faint sources or those outside the ROI are held fixed at the 3FGL values. The results are shown in Table 2. The highestenergy bands in which a significant detection $(\mathrm{TS}>15)$ was made are $29-75 \mathrm{GeV}, 75-194 \mathrm{GeV}$, and $75-194 \mathrm{GeV}$ for J0022, J0630, and J0811, respectively (see Figure 3). We then derive the SEDs using the best-fit power-law model and show the inferred spectrum in Figure 3, where the TS is greater than 15 for each data point. We performed the analysis using different ROI sizes, finding consistent results. In Figure 3 we show the results obtained for the $5^{\circ}$ extraction because it gives the highest TS value.

We show the broadband SEDs in Figure 3. A noncontemporaneous broadband SED for J0630 with sparser X-ray and gamma-ray data has been previously reported (Ghisellini et al. 2012; Padovani et al. 2012); the results are broadly similar to our measurements.

\subsection{SED Modeling}

We use the one-zone synchro-Compton model of Boettcher et al. (1997, hereafter B97) to model the SEDs of the sources. The code evolves a spherical blob of electron/positron plasma with a power-law injected energy distribution, following the $e^{+} / e^{-}$population over $10^{7} \mathrm{~s}\left(t_{\mathrm{evol}}\right)$, assuming that the particle energy loss is dominated by radiative cooling as the blob zone flows along a jet axis. As blobs are continuously injected, the emission zone forms a cylindrical shape (i.e., jet) elongated along the jet axis $\left(l=c t_{\text {evol }}=3 \times 10^{17} \mathrm{~cm}\right)$, and the timeintegrated spectrum determines the jet emission. The effect of pair absorption is calculated and included in the model. The full model has 16 parameters, including those for disk and BLR emission; to simplify, we start with standard BL Lac assumption that self-Compton emission dominates, so the seed photons from BLR and disk are negligible. The seven remaining parameters we adjust are the low-energy and highenergy cutoffs $\left(\gamma_{\text {min,max }}^{\prime}\right)$ and spectral index of the power-law electron distribution $\left(p_{1}\right)$, the magnetic field strength $(B)$, the bulk Lorentz factor of the jet $(\Gamma)$ (this is done for a fixed viewing angle $\theta_{\mathrm{v}}$, hence equivalent to adjusting the Doppler factor $\left.\delta_{\mathrm{D}}\right)$, and the blob rest-frame size $\left(R_{b}^{\prime}\right)$ and electron density $\left(n_{e}\right)$, which serve to normalize the total flux. This model has also been used to model SEDs of other blazars (e.g., Hartman et al. 2001; Romani 2006).

We use the following steps to find best-fit SED parameters: (1) adjust the parameters to visually match the SED for initial values, (2) vary each individual parameter over a range (a factor of $\sim 2$ initially and decreased with iterations) with 10 grid points while holding the other parameters fixed, (3) find the parameter value that provides the minimum $\chi^{2}$, (4) update the 
Table 3

Best-fit Parameters for the SSC Model of B97 with Single Power-law Injection

\begin{tabular}{|c|c|c|c|c|}
\hline Parameter & Symbol & 3FGL J0022.1-1855 & 3FGL J0630.9-2406 & 3FGL J0811.2-7529 \\
\hline Redshift & $z$ & 0.774 & $>1.239$ & 0.689 \\
\hline Doppler factor & $\delta_{\mathrm{D}}$ & 19 & 71 & 33 \\
\hline Bulk Lorentz factor & $\Gamma$ & $>9.6$ & $>35.3$ & $>16.5$ \\
\hline Viewing angle (deg.) & $\theta_{v}$ & $<3.0$ & $<0.81$ & $<1.74$ \\
\hline Magnetic field (mG) & $B$ & 60 & 1016 & 7 \\
\hline Comoving radius of blob $(\mathrm{cm})$ & $R_{b}^{\prime}$ & $1.12 \times 10^{14}$ & $1.78 \times 10^{13}$ & $1.52 \times 10^{14}$ \\
\hline Effective radius of the blob $(\mathrm{cm})$ & $R_{\mathrm{E}}^{\prime}=\left(3 R_{b}^{\prime 2} t_{\mathrm{evol}} c / 4\right)^{1 / 3}$ & $1.4 \times 10^{15}$ & $1.9 \times 10^{14}$ & $1.7 \times 10^{15}$ \\
\hline Initial electron spectral index & $p_{1}$ & 3.14 & 4.26 & 3.19 \\
\hline Initial minimum electron Lorentz factor & $\gamma_{\min }^{\prime}$ & $2.88 \times 10^{4}$ & $1.41 \times 10^{4}$ & $1.18 \times 10^{4}$ \\
\hline Initial maximum electron Lorentz factor & $\gamma_{\max }^{\prime}$ & $1.5 \times 10^{6}$ & $2.7 \times 10^{7}$ & $3 \times 10^{7}$ \\
\hline Injected particle luminosity $\left(\mathrm{erg} \mathrm{s}^{-1}\right)^{\mathrm{a}}$ & $L_{\text {inj }}$ & $9 \times 10^{42}$ & $7 \times 10^{41}$ & $8 \times 10^{42}$ \\
\hline$\chi^{2} /$ dof & $\cdots$ & $151.1 / 122$ & $186 / 140$ & $128.5 / 94$ \\
\hline Synchrotron peak frequency $(\mathrm{Hz})^{\mathrm{b}}$ & $\nu_{\mathrm{pk}}^{\mathrm{sy}}$ & $5.6 \times 10^{14}$ & $1.5 \times 10^{15}$ & $5.8 \times 10^{14}$ \\
\hline Synchrotron peak luminosity $\left(\mathrm{erg} \mathrm{s}^{-1}\right)^{\mathrm{b}}$ & $L_{\mathrm{pk}}^{\mathrm{sy}}$ & $4.6 \times 10^{45}$ & $6.7 \times 10^{46}$ & $5.1 \times 10^{45}$ \\
\hline Compton dominance & $\mathrm{CD}$ & 1.2 & 1.4 & 2.1 \\
\hline
\end{tabular}

Notes.

${ }^{a}$ Energy injected into the jet in the jet rest frame (see Böttcher \& Chiang 2002).

${ }^{\mathrm{b}}$ Quantities in the observer frame. The luminosity quoted is that inferred assuming isotropic emission.

parameter found in step (3) with the best-fit value, and (5) repeat (2)-(4) until the fit does not improve any more. Because the X-ray spectra are so well described by a simple power law, we initially identify their spectra with the synchrotron emission of a cooled electron population, strongly constraining the fit parameter set. We do not include the highest-energy $(\gtrsim 40$ $\mathrm{GeV}$ ) LAT points in the initial fits as we will use them later for EBL constraints, as done by Domínguez et al. (2013). We update only one parameter in each iteration, although we vary all seven parameters. We present the best-fit parameters in Table 3 . We also measured $\nu_{\mathrm{pk}}^{\mathrm{sy}}, L_{\mathrm{pk}}^{\mathrm{sy}}$, and CD using the best-fit SED model, and we present them in Table 3.

In the model, $\Gamma$ and $\theta_{\mathrm{v}}$ appear only in combination through the Doppler factor $\delta_{\mathrm{D}}=[\Gamma(1-\beta \mu)]^{-1}$, where $\beta=\sqrt{1-1 / \Gamma^{2}}$ and $\mu=\cos \left(\theta_{\mathrm{v}}\right)$. Hence, the model determines only $\delta_{\mathrm{D}}$ unless one has external constraints on one of $\Gamma$ or $\theta_{\mathrm{v}}$. Therefore, for a given $\delta_{\mathrm{D}}$, only lower and upper limits for $\Gamma$ and $\theta_{\mathrm{v}}$ can be inferred, also given in Table 3 .

While the procedure above converges well to a local minimum, there is always a risk that quite distinct solutions could provide better fits. The high dimensionality of the fit space, plus the incomplete SED coverage, makes it difficult to locate such minima. To aid our exploration of parameter space, we used the initial scans to define the covariance between the various quantities. We find that simple power-law codependencies capture most of the covariance trend around the fit minimum. We fit an amplitude and slope for each parameter pair. Thus, by varying one control parameter, say $B$, and then setting the others to the covariance-predicted values, we can take larger steps without wandering too far from the $\chi^{2}$ minimum surface. For each such trial solution, we then compute small test grids to rapidly converge to the local minimum (with the control parameter held fixed). In this way we explored the minima connected to the "best fit" solution tabulated above. This gave us larger ranges for "acceptable" (i.e., null hypothesis probability $p>0.01$ ) solutions. For example, for J0630, acceptable solutions were found for
$0.3 \mathrm{G}<B<3 \mathrm{G}$, although all were poorer fits than the best solution (Table 3).

We note that the J0811 flux in the Fermi band may be lower by $\sim 20 \%$ than is used in the modeling if we remove $\mathrm{J} 0810$ contamination (see Section 2.4). We therefore performed a Fermi data analysis including J0810 and constructed a new SED of J0811. We modeled the new SED as described above and found that significant changes need to be made only for parameters related to high-energy normalization, and our conclusion on EBL constraints below remains the same.

\subsection{EBL Constraints}

We have been careful not to use the highest-energy LAT points in the SSC SED fits, although we see that all models overpredict the high-energy LAT flux. We now apply EBL models to the data and calculate $\chi^{2}$ with and without EBL models, showing the results in Table 4 (see also Figures 3-5). Note that we used all the SED data, including those $>40 \mathrm{GeV}$ here. Not unexpectedly, EBL absorption provides no significant improvement to the fits of the lower-redshift sources J0022 and J0811. However, we see clear improvements $\left(\Delta \chi^{2} \sim 10\right.$ corresponding to $\sim 5 \sigma$ ) for J0630. Only the high-UV model provides no improvement. The $\chi^{2}$ decrease is similar for the more conventional models.

Since the redshift measurement for J0630 is only a lower limit, we attempted to fit $z$ in the EBL model fits. Allowing one more free parameter (holding the other parameters fixed) improves the fit in general, but the improvement is small, except for the case of the disfavored models. For all models, the best-fit $z$ is less than the spectroscopic lower limit, although this is within errors for the best-fit models. Accordingly, we hold $z$ fixed at 1.239 .

Although the LAT observations continue, unless there is a strong flare, we are unlikely to greatly improve the J0630 EBL constraints without going to higher energy. This will be challenging with the present and future-generation air Cerenkov telescopes; we predict an absorbed $200 \mathrm{GeV}$ energy flux of 
Table 4

Best-fit $\chi^{2}$ Values for the EBL Models Tested in This Work

\begin{tabular}{lcccc}
\hline \hline Model & J0022 & J0630 & J0811 & References \\
\hline No EBL & 151.1 & 197.4 & 128.9 & $\ldots$ \\
Domínguez & 151.1 & 186.2 & 129.6 & $(1)$ \\
Franceshini & 151.1 & 186.2 & 129.6 & $(2)$ \\
Gilmore Fiducial & 151.0 & 189.2 & 130.0 & $(3)$ \\
Gilmore Fixed & 151.1 & 186.5 & 129.6 & $(3)$ \\
Helgason & 151.1 & 186.3 & 129.5 & $(4)$ \\
Kneiske04 best fit & 151.1 & 191.4 & 130.6 & $(5)$ \\
Kneiske \& Dole & 151.1 & 187.4 & 129.8 & $(6)$ \\
Kneiske high UV & 150.3 & 205.1 & 132.8 & $(5)$ \\
Stecker high opac. & 151.0 & 194.0 & 131.6 & $(7)$ \\
Stecker low opac. & 151.0 & 187.4 & 130.2 & $(7)$ \\
Finke "C" & 151.1 & 187.0 & 129.7 & $(8)$ \\
\hline
\end{tabular}

References: (1) Domínguez et al. (2011), (2) Franceschini et al. (2008), (3) Gilmore et al. (2012), (4) Helgason \& Kashlinsky (2012), (5) Kneiske et al. (2004), (6) Kneiske \& Dole (2010), (7) Stecker et al. (2012), (8) Finke et al. (2010).

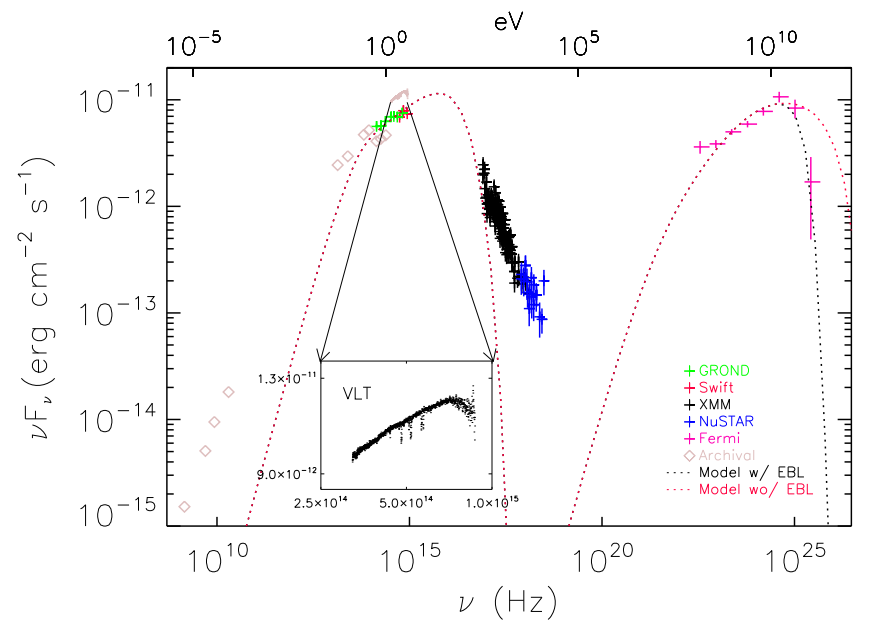

Figure 4. SED model fit with (black dotted line) and without (red dotted line) the EBL absorption model (Finke "C" in Table 4) for the J0630 data with a hard injection spectrum. The parameters for this model are $\delta_{\mathrm{D}}=73$, $\theta_{\mathrm{v}}=0.74, \quad B=10 \mathrm{mG}, \quad R_{\mathrm{b}}^{\prime}=2 \times 10^{14} \mathrm{~cm}, \quad p_{1}=2.35, \quad \gamma_{\min }=5 \times 10^{3}$, and $\gamma_{\max }=2 \times 10^{5}$.

$\nu F_{\nu} \sim 4 \times 10^{-14} \mathrm{erg} \mathrm{cm}^{-2} \mathrm{~s}^{-1}$, which is an order of magnitude lower than the 5- $\sigma$ sensitivity of the Cherenkov Telescope Array. ${ }^{58}$ Further LAT study of other high-redshift BL Lacs can certainly probe the EBL evolution at $z>1.5$.

\subsection{Alternative Fits}

The best-fit parameters for our BL Lacs are unusual with steep $p_{1}>3$ injection spectra. J0630 is the most extreme, with $p_{1} \approx 4.3$ and a strong $\sim 1 \mathrm{G}$ magnetic field. The excellent power-law fits to the $X M M-N u S T A R$ X-ray data drive these values. We have attempted to fit J0630 with more conventional $2<p_{1}<3$ indices, but such models are always strongly excluded by the X-ray spectral points. The only option is to remove the X-ray points from the fits and assign them to an additional, unmodeled component. Then excellent fits to the rest of the SED with more conventional, lower $p_{1}$ and $B$ values can be obtained, an example of which is shown in Figure 4.

\footnotetext{
58 https://portal.cta-observatory.org/Pages/Home.aspx
}

The synchrotron peak energy is higher (consonant with the high source power), and the X-rays are underpredicted; the observed spectrum is an additional, soft component. This soft component, if produced by synchrotron emission, can be generated by an electron distribution with $\gamma_{\text {min }}^{\prime}>4 \times 10^{4}$, $\gamma_{\max }^{\prime}=5 \times 10^{6}, \quad p_{1}=4.1$, and a small electron density $\sim 10^{-1} \mathrm{~cm}^{-3}$ in order not to overproduce the optical and the Compton emission.

We are focused on the LAT band fit, so it is interesting to see that this model has a very similar cutoff to that of Figure 3(b), requiring a similar EBL absorption. The $\chi^{2}$ values (18 data points ignoring the X-ray data) are 62 and 86 with and without the EBL absorption, respectively. Evidently, inverse-Compton emission from the X-ray component, if any, is in the highly absorbed teraelectronvolt band. We can speculate that the soft X-ray component arises in a different zone of the jet (e.g., Marscher 2014), arguably with large $B$ and a steep, highly cooled spectrum. Whether this is connected to the apparently different absorption for this component is unclear.

If we allow an additional X-ray-emitting component, we might also consider a more complex injection model (Finke et al. 2008, hereafter FDB08). We try an electron distribution that is a broken power law or a log parabola. To compare parameters, we fit to this model by first choosing a variability timescale and then adjusting the other parameters $\left(\delta_{\mathrm{D}}, B\right.$, and the electron distribution) until a good fit is obtained. We assumed $t_{v}=10^{5} \mathrm{~s}$, which is consistent with the timescale for the optical flux variability in J0630 ( $t_{v} \lesssim 3$ days). The broken power-law model is always more satisfactory than the logparabola version, and we show the best-fit parameters for our three BL Lacs in Table 5. It is interesting to compare this to our cooling model fits. In particular, the power law breaks strongly to large $p_{2}$ values. This is imposed by fiat here, but the drive to such a large break is difficult to accommodate in self-consistent cooling and can require large magnetic field strengths (Table 3). We conclude that if conventional $p_{1} \sim 2-3$ electron injection spectra are adopted, we will always require an additional steep component not easily achieved by radiative cooling.

We have noted that the $>\mathrm{GeV}$ LAT spectrum is not affected by this extra electron component (and thus our EBL conclusions for J0630 are robust). However, this is in the context of SSC models. Ghisellini et al. (2012) and Padovani et al. (2012) noted that HSP BL Lacs can also have low-level disk or BLR emission, overwhelmed by (and invisible behind) the jet synchrotron component along the Earth line of sight, yet providing substantial seed photons for Compton upscatter. These may have a significant impact on the high-energy hump of the SED (blue FSRQ model; Ghisellini et al. 2012; Padovani et al. 2012). Thus, we explore the B97 model for J0630 with a disk component (orders of magnitude fainter than the baseline synchrotron emission) that can produce additional Compton emission at $\sim 10^{24}-10^{26} \mathrm{~Hz}$ (Figure 5). We assume a small BL covering fraction given the strong limits on broad-line equivalent widths (Shaw et al. 2013).

In Figure 5(a), we add disk EC emission to the model of Figure 3 with a soft $\left(p_{1}=4.26\right)$ injection spectrum. The strong constraint of the X-ray data precludes any large change in the SSC component. We find that the additional EC emission contributes primarily at high LAT energies. The net effect is to underproduce the low-energy gamma rays, leading to an excessively hard LAT spectrum while not significantly changing the high-energy spectral shape. Thus the EC is not 


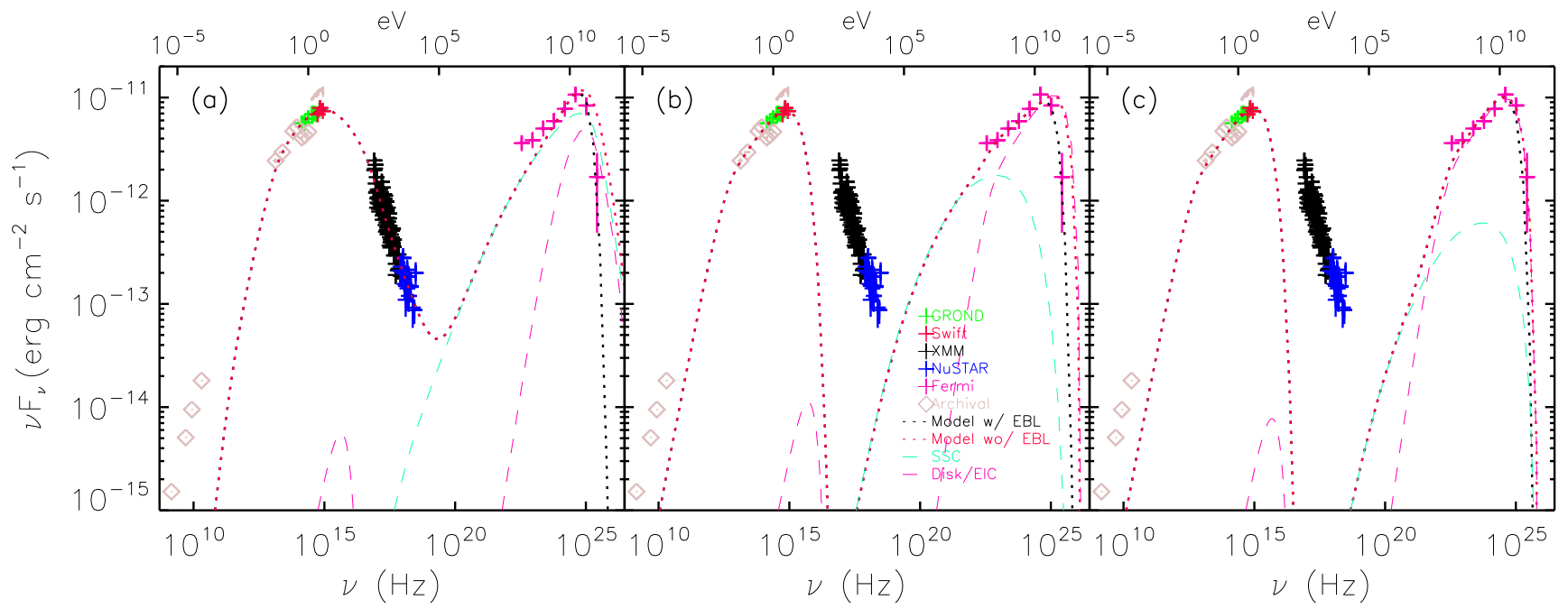

Figure 5. SED models with the disk component for J0630: (a) a model with the disk component added to the baseline synchrotron+SSC model in Figure 3(b); (b) similar to (a) but baseline model is that in Figure 4; (c) same as (b) with larger $B$ and lower $\gamma_{\max }$. The model parameters are further adjusted from the baseline ones to match the SED. The EBL model we used for the plot is the "Finke C" model in Table 4. See text for more details.

statistically demanded by this model, but even if EC is added, significant EBL absorption should be present; the improvement of the fit when the EBL models in Table 4 are included is typically $\Delta \chi^{2} \sim 20$.

The addition of the disk/EC component to the model in Figure 4 (hard injection spectrum) provides more flexibility since we do not need to match the X-ray spectrum, having assumed above that the X-ray emission in this model is from a different region than the peak jet emission. In this case, the shape of the SSC component can be adjusted to match the lowenergy gamma-ray data, and the EC emission accounts for the higher energy data (Figure 5(b)); this model reproduces the optical/UV and gamma-ray data better than the baseline model (Figure 4) does. Nevertheless, the effect of EBL absorption is clearly visible in Figure 5(b), and including the EBL models improves the fit by $\Delta \chi^{2} \sim 40$.

It may be imagined that the sharp drop above $10^{25} \mathrm{~Hz}$ in the unabsorbed model (dashed magenta line in Figure 5(b)) may be able to reproduce the sharp drop in the SED without a visible effect of the EBL absorption if the peak frequency of the EC component can be lowered. This can be done by lowering $\gamma_{\text {max }}^{\prime}$, but merely adjusting $\gamma_{\max }^{\prime}$ will damage the goodness of fit in the optical/UV band. However, by adjusting $B, \gamma_{\max }^{\prime}$, and $\Gamma$ $\left(\delta_{\mathrm{D}}\right)$, lowering only $\nu_{\mathrm{pk}}^{\mathrm{IC}}$ without affecting $\nu_{\mathrm{pk}}^{\mathrm{sy}}$ is possible since the latter is $\propto \Gamma B \gamma_{\max }^{\prime 2}$ while the former is $\propto \Gamma^{2} \gamma_{\max }^{\prime 2}$. We first adjust $B$ (decrease) and $\gamma_{\max }^{\prime}$ (increase), and we find that $\nu_{\mathrm{pk}}^{\mathrm{sy}}$ is also lowered in this case, owing to stronger cooling caused by the stronger magnetic field strength. So we lowered $\Gamma$ and adjusted $B$ and $\gamma_{\max }^{\prime}$. In this way, we were able to match the steep fall in the SED at $\gtrsim 10^{25} \mathrm{GHz}$ without invoking EBL absorption (Figure 5(c)). For this model, we use $B=15 \mathrm{mG}$, $\gamma_{\text {max }}^{\prime}=8 \times 10^{4}$, and $\delta_{\mathrm{D}}=27$ (corresponding to $\Gamma>14$ and $\theta_{v}<2$. 1 ). In this case, as we intended, the fit is better when the EBL absorption is not considered; the EBL effect makes the model underpredict the data, and including the EBL models increases $\chi^{2}$ by $\sim 3$ typically. Note that for the models in Figures 5(b) and (c) we assumed that there is a sharp highenergy cutoff in the synchrotron emission. However, if such a sharp cutoff does not exist, the high-frequency SSC/EC component should be enhanced, perhaps similar to that in Figure 5(a), requiring the EBL absorption.

Note that we can also add BLR-reflected disk photons to this model (see Romani 2006, for example). The EC emission of the reflected photons only appears at higher frequencies than the direct disk component and thus suffers from severe EBL absorption. Therefore, we do not consider this component here.

\section{DISCUSSION AND CONCLUSIONS}

We constructed broadband SEDs for three high-redshift BL Lac objects, J0022, J0630, and J0811, using nearly contemporaneous observations in the optical to X-ray band. Studying the LAT data, we conclude that the variability on day-to-year timescales is fairly low for these three systems. This allows us to use the 6-year (mission-averaged) LAT spectrum in forming our SED. We fit the SEDs with a synchrotron/Compton model to infer physical properties of the sources.

Interestingly, Figure 3 shows that there is a trend for highflux optical states to be spectrally harder. Similar trends have been seen in other blazars (e.g., Zhang et al. 2012). Our contemporaneous data (and SED modeling) are for the low, relatively quiescent state. We lack the broadband high-state coverage to study the physical properties, imposing this variation via separate SED fits. Still, if the variation (increase in $L_{\mathrm{pk}}^{\mathrm{sy}}$ and $\nu_{\mathrm{pk}}^{\mathrm{sy}}$ ) were produced by an increase in the external photon field, one expects $\nu_{\mathrm{pk}}^{\mathrm{sy}}$ to decrease because the jet particles should cool more efficiently. This is not observed, so we infer that the variation is likely produced in the injection particle spectrum or in the jet blob flow (e.g., increase in $\delta_{\mathrm{D}}$ ) and $B$ field. This suggests correlated optical gigaelectronvolt variability, which may be too weak for the LAT to detect.

The basic B97 modeling constrains the emission parameters well under the assumptions of pure SSC emission and radiative cooling of the injected electrons (Figure 3). The SED fits assuming only the assigned statistical errors is adequate (probabilities $p r=10^{-2}-10^{-3}$ ). However, there are almost certainly additional systematic errors, including extinction uncertainty and interinstrument calibrations. For example, increasing the measurement uncertainties by $5 \%$ (all of the SED data points) makes the fit acceptable, with $p r \sim 10 \%$. 
Table 5

Best-fit Parameters of the FDB08 Model

\begin{tabular}{|c|c|c|c|c|}
\hline Parameter & Symbol & J0022.1-1855 & 3FGL J0630.9-2406 & 3FGL J0811.2-7529 \\
\hline Redshift & $z$ & 0.774 & $>1.239$ & 0.689 \\
\hline Doppler factor & $\delta_{\mathrm{D}}$ & 29 & 110 & 49 \\
\hline Magnetic field (mG) & $B$ & 37 & 4.7 & 7.9 \\
\hline Variability timescale (s) & $t_{v}$ & $10^{5}$ & $10^{5}$ & $10^{5}$ \\
\hline Comoving radius of blob $(\mathrm{cm})$ & $R_{b}^{\prime}$ & $4.9 \times 10^{16}$ & $1.5 \times 10^{17}$ & $8.7 \times 10^{16}$ \\
\hline Lower-energy electron spectral index & $p_{1}$ & 2.5 & 2.4 & 2.6 \\
\hline High-energy electron spectral index & $p_{2}$ & 4.0 & 4.5 & 4.0 \\
\hline Minimum electron Lorentz factor & $\gamma_{\min }^{\prime}$ & $6 \times 10^{3}$ & $10^{3}$ & $3 \times 10^{3}$ \\
\hline Break electron Lorentz factor & $\gamma_{\text {brk }}^{\prime}$ & $3.9 \times 10^{4}$ & $6.9 \times 10^{4}$ & $4.9 \times 10^{4}$ \\
\hline Maximum electron Lorentz factor & $\gamma_{\max }^{\prime}$ & $3.0 \times 10^{6}$ & $3.0 \times 10^{6}$ & $6 \times 10^{6}$ \\
\hline
\end{tabular}

The SED parameters are, however, somewhat unusual, giving particularly soft injection spectra, with $p_{1}$ well above that expected for relativistic shock acceleration: $p_{1} \sim 2-2.5$. For J0022 and J0811, higher $p_{1}$ are required because of the flat SED $\left(\alpha=0\right.$ in $\left.\nu F_{\nu} \propto \nu^{\alpha}\right)$ in the optical band, which requires $p_{1} \sim 3$. If we identify this with the cooled spectrum, allowing harder injection, then we cannot accommodate the steeper $\mathrm{X}$-ray spectrum since radiative cooling produces only a $\Delta \alpha=0.5$ break (if the electrons were in the Klein-Nishina regime, the break would be even weaker). Similarly, matching the J0630 optical spectrum $(\alpha \sim 0.2)$ and X-ray spectrum $(\alpha \sim-1)$ is not possible if we let the electrons cool with the break between the optical and the X-ray bands (Figure 4). Thus we are forced to very steep injection spectra if the X-rays are produced by the same population as the optical emission. This conclusion is supported by fitting with more complex heuristic electron spectra (FDB08 model). With such models, we can avoid the very high magnetic field strength required for J0630 to implement the rapid X-ray cooling and use lower $10 \mathrm{mG}$ fields.

The minimum electron energies for the sources are rather high. While these values are not unusual when compared to those in other works (e.g., Tavecchio et al. 2010), it is not clear what environments or conditions are required in the acceleration site to achieve such high minimum electron energies, and further investigations are needed to tell whether or not such values are realistic. Note that we do not use the equipartition magnetic field strength in our modeling, and the particle energy is much larger than the magnetic energy in our models. In particular, the inferred magnetic field strength for J0811 is very low compared to those for previously studied BL Lacs (see Finke et al. 2008; Tavecchio et al. 2010; Zhang et al. 2012, for example), although there are several objects in the literature with lower inferred $B$ (and lower magnetic-to-particle-energy ratio). As we already noted (Section 3.3), it may be possible to find another solution with lower $\gamma_{\min }$ and higher $B$. Covering the SED more completely will help us to infer the parameters more precisely. Nevertheless, the SED at the high-energy end is primarily determined by the X-ray spectrum in our model, and thus our conclusion on the EBL would not change.

By excluding the X-rays from the SED fit, we can indeed accommodate lower injection $p_{1}$, but the cost is that the $\mathrm{X}$-ray must be an independent, steep spectrum component. Heuristic modeling with inferred stationary $e^{+} e^{-}$spectra confirm that a very steep population is needed to model the X-ray component. Thus a simple, single-zone SSC model with typical particle acceleration spectra is inadequate. The additional ingredient may be a separate, steep cooled jet population for the X-ray emission. There is some indication of separate X-ray/optical components seen in the different absorption columns inferred from the two bands for J0630. However, other effects (e.g., adiabatic expansion cooling) may also be relevant.

We find that the $\gtrsim 100 \mathrm{GeV}$ LAT points for our highestredshift source J0630 are generally significantly overpredicted by our SED models, and we take this to be strong evidence of the effect of EBL absorption. Standard EBL models do a good job of producing the observed spectral cutoff, but high-UV models are not satisfactory (see also Ackermann et al. 2012; H. E.S.S. Collaboration et al. 2013). This conclusion is fairly robust, and EBL absorption is still required if we allow the observed X-ray emission to be a separate jet component. Introduction of EC components from faint (unobserved) disk emission affects the shape of the LAT spectrum. In general, the harder EC spectrum does not match the LAT data, and it is difficult to arrange the components to mimic the high-energy cutoff; EBL absorption is still preferred unless the synchrotron cutoff is extraordinarily sharp. We can approximate this with an abrupt cutoff in the electron energy distribution (Figure 5(c)), but such a sharp feature is unlikely to be realized in physical acceleration models. Note that the effects of EBL absorption are not clearly visible in the low-redshift sources as expected in EBL models; the optical depth at $50 \mathrm{GeV}$ for $z=0.7$ is only 0.08 as estimated with the Domínguez model in Table 4.

We conclude with a few comments about the place of our sources in the BL Lac population. Our objects are luminous with high $\nu_{\mathrm{pk}}^{\mathrm{sy}}$, so it is natural to consider their relation to the "blazar sequence." In Figure 6, we plot $L_{\mathrm{pk}}^{\mathrm{sy}}$ and CD (Finke 2013) versus $\nu_{\mathrm{pk}}^{\mathrm{sy}}$ (in the source rest frame) for blazars from the 3LAC sample, including our three sources. The general trend is commonly attributed to the effect of an increased external photon field (e.g., from the BLR or disk) for blazars with lower $\nu_{\mathrm{pk}}^{\mathrm{sy}}$ and magnetic field strength (e.g., Ghisellini et al. 1998; Finke 2013). Our three sources are HSPs or ISPs but are relatively close to the ISP border. They show higher $L_{\mathrm{pk}}^{\mathrm{sy}}$ and higher $\mathrm{CD}$ than the general population, but only J0630 is a true outlier in the $L_{\mathrm{pk}}^{\mathrm{sy}}$ plot. In fact, with the quiescent-state SED assembled here, it is somewhat less extreme than in previous studies. Still, as one of the four high-redshift BL Lacs called out by Padovani et al. (2012), it does present some challenges to the simple blazar sequence. A more complete study of the high-redshift LAT BL Lacs is needed to see if such sources are a robust population and thus conflict with the blazar sequence correlation. If so, sources such 

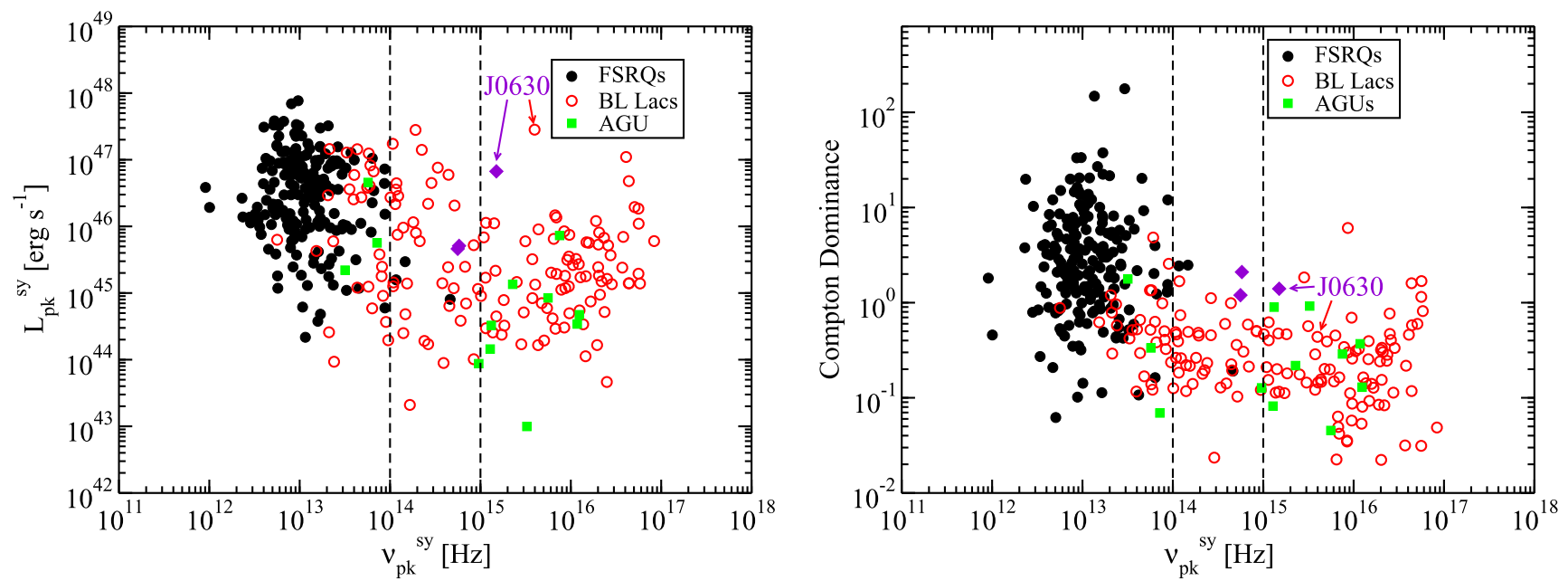

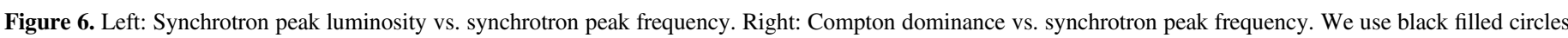

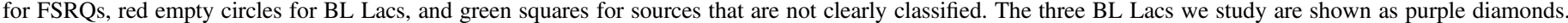
Note that the red circle for J0630 shows the position of the source reported in a previous study (Finke 2013).

as J0630 may be FSRQs viewed very close to the jet axis $\left(\theta_{\mathrm{v}}<0.81 \mathrm{deg}\right.$; Table 3) such that the disk/BLR emission is overwhelmed by the beamed jet emission. A detailed study along the lines of the blue FSRQ model (Ghisellini et al. 2012) using our high-quality contemporaneous SEDs would be quite interesting.

Since $L_{\mathrm{pk}}^{\mathrm{sy}}$ is redshift dependent, it is more subject to selection effects in a survey study. Thus it is argued (e.g., Finke 2013) that CD is a more robust classifier of the blazar status, being redshift independent (although still sensitive to viewing angle effects if EC components contribute). In Figure 6 right (see Finke 2013 for more details), we see that our three sources lie near the upper edge of the HSP population. These are highly Compton-dominated sources but not really distinct from the rest of the HSP population. Since our three sources, and the other high-peak/high-power BL Lacs, still follow a general correlation in this plot, it suggests that the blazar sequence scenario may still be robust to inclusion of highpower, high-redshift BL Lacs.

Nonetheless, the Doppler factors $\left(\delta_{\mathrm{D}}\right)$ of these three sources are fairly large. Following the cosmic evolution, Ajello et al. (2014) inferred the distribution of the Lorentz factor $(\Gamma)$ and the viewing angle $\left(\theta_{\mathrm{v}}\right)$ for the LAT blazar population. We note that the distribution for $\theta_{\mathrm{v}}$ derived by Ajello et al. (2014) (their Figure 9) is broad, and the values we inferred with the models (Tables 3) are not exceptional. However, the best-fit Lorentz factors are very high considering the power-law distribution with the slope $k=-2.03 \pm 0.70$ for BL Lacs (Ajello et al. 2014). In order for the chance probability of having $\Gamma>35.3$ (for J0630) to be greater than $1 \%, k$ should be greater than -2.49 . So perhaps our sources do represent a highvelocity, tightly beamed wing of the BL Lac population, and their unusual properties are due to beaming effects.

Whether or not BL Lacs at $z>1$ contradict our present picture of the source evolution, our SED measurements, particularly those for J0630, show that these sources can be a powerful probe of the EBL and its evolution. We anticipate more striking EBL constraints as we push to the peak of cosmic star formation via further study of high-redshift Fermi-detected BL Lacs.
This work was supported under NASA Contract No. NNG08FD60C and made use of data from the NUSTAR mission, a project led by the California Institute of Technology, managed by the Jet Propulsion Laboratory, and funded by the National Aeronautics and Space Administration. We thank the NuSTAR Operations, Software, and Calibration teams for support with the execution and analysis of these observations. This research has made use of the NUSTAR Data Analysis Software (NuSTARDAS), jointly developed by the ASI Science Data Center (ASDC, Italy) and the California Institute of Technology (USA).

The Fermi LAT Collaboration acknowledges generous ongoing support from a number of agencies and institutes that have supported both the development and the operation of the LAT as well as scientific data analysis. These include the National Aeronautics and Space Administration and the Department of Energy in the United States, the Commissariat à l'Energie Atomique and the Centre National de la Recherche Scientifique/Institut National de Physique Nucléaire et de Physique des Particules in France, the Agenzia Spaziale Italiana and the Istituto Nazionale di Fisica Nucleare in Italy, the Ministry of Education, Culture, Sports, Science and Technology (MEXT), the High Energy Accelerator Research Organization (KEK), and the Japan Aerospace Exploration Agency (JAXA) in Japan, and the K. A. Wallenberg Foundation, the Swedish Research Council, and the Swedish National Space Board in Sweden.

Additional support for science analysis during the operations phase is gratefully acknowledged from the Istituto Nazionale di Astrofisica in Italy and the Centre National d'Études Spatiales in France.

H.A. acknowledges support provided by the NASAsponsored Fermi Contract NAS5-00147 and by the Kavli Institute for Particle Astrophysics and Cosmology (KIPAC). Part of the funding for GROND (both hardware and personnel) was generously granted from the Leibniz Prize to Prof. G. Hasinger (DFG grant HA 1850/28-1).

\section{REFERENCES}

Abdo, A. A., Ackermann, M., Agudo, I., et al. 2010, ApJ, 716, 30 
Acero, F., Ackermann, M., Ajello, M., et al. 2015, ApJS, 218, 23

Ackermann, M., Ajello, M., Allafort, A., et al. 2011, ApJ, 743, 171

Ackermann, M., Ajello, M., Allafort, A., et al. 2012, Sci, 338, 1190

Ajello, M., Romani, R. W., Gasparrini, D., et al. 2014, ApJ, 780, 73

Aleksić, J., Ansoldi, S., Antonelli, L. A., et al. 2015, A\&A, 573, A50

Atwood, W., Albert, A., Baldini, L., et al. 2013, arXiv:1303.3514

Atwood, W. B., Abdo, A. A., Ackermann, M., et al. 2009, ApJ, 697, 1071

Beckmann, V., Engels, D., Bade, N., \& Wucknitz, O. 2003, A\&A, 401, 927

Blandford, R. D., \& Rees, M. J. 1978, PhyS, 17, 265

Boettcher, M., Mause, H., \& Schlickeiser, R. 1997, A\&A, 324, 395

Böttcher, M., \& Chiang, J. 2002, ApJ, 581, 127

Böttcher, M., \& Dermer, C. D. 2002, ApJ, 564, 86

Cavaliere, A., \& D'Elia, V. 2002, ApJ, 571, 226

Cohen, D. P., Romani, R. W., Filippenko, A. V., et al. 2014, ApJ, 797, 137

D’Abrusco, R., Massaro, F., Ajello, M., et al. 2012, ApJ, 748, 68

Domínguez, A., Finke, J. D., Prada, F., et al. 2013, ApJ, 770, 77

Domínguez, A., Primack, J. R., Rosario, D. J., et al. 2011, MNRAS, 410, 2556

Finke, J. D. 2013, ApJ, 763, 134

Finke, J. D., Dermer, C. D., \& Böttcher, M. 2008, ApJ, 686, 181

Finke, J. D., Razzaque, S., \& Dermer, C. D. 2010, ApJ, 712, 238

Foight, D., Guver, T., Ozel, F., \& Slane, P. 2015, arXiv:1504.07274

Fossati, G., Maraschi, L., Celotti, A., Comastri, A., \& Ghisellini, G. 1998, MNRAS, 299, 433

Franceschini, A., Rodighiero, G., \& Vaccari, M. 2008, A\&A, 487, 837

Gehrels, N., Chincarini, G., Giommi, P., et al. 2004, ApJ, 611, 1005

Ghisellini, G., Celotti, A., Fossati, G., Maraschi, L., \& Comastri, A. 1998 MNRAS, 301, 451

Ghisellini, G., Tavecchio, F., Foschini, L., et al. 2010, MNRAS, 402, 497

Ghisellini, G., Tavecchio, F., Foschini, L., et al. 2012, MNRAS, 425, 1371

Gilmore, R. C., Somerville, R. S., Primack, J. R., \& Domínguez, A. 2012, MNRAS, 422, 3189

Giommi, P., Padovani, P., Polenta, G., et al. 2012, MNRAS, 420, 2899

Gorenstein, P. 1975, ApJ, 198, 95

Greiner, J., Bornemann, W., Clemens, C., et al. 2008, PASP, 120, 405
Harrison, F. A., Craig, W. W., Christensen, F. E., et al. 2013, ApJ, 770, 103

Hartman, R. C., Böttcher, M., Aldering, G., et al. 2001, ApJ, 553, 683

Helgason, K., \& Kashlinsky, A. 2012, ApJL, 758, L13

H.E.S.S. Collaboration, Abramowski, A., Acero, F., et al. 2013, A\&A, 550, A4

Jansen, F., Lumb, D., Altieri, B., et al. 2001, A\&A, 365, L1

Kneiske, T. M., Bretz, T., Mannheim, K., \& Hartmann, D. H. 2004, A\&A, 413, 807

Kneiske, T. M., \& Dole, H. 2010, A\&A, 515, A19

Komatsu, E., Smith, K. M., Dunkley, J., et al. 2011, ApJS, 192, 18

Krühler, T., Küpcü Yoldaş, A., Greiner, J., et al. 2008, ApJ, 685, 376

Landt, H., Padovani, P., Perlman, E. S., \& Giommi, P. 2004, MNRAS, 351,83

Mannheim, K., \& Biermann, P. L. 1992, A\&A, 253, L21

Marcha, M. J. M., Browne, I. W. A., Impey, C. D., \& Smith, P. S. 1996, MNRAS, 281, 425

Marscher, A. P. 2014, ApJ, 780, 87

Nolan, P. L., Abdo, A. A., Ackermann, M., et al. 2012, ApJS, 199, 31

Padovani, P., \& Giommi, P. 1995, ApJ, 444, 567

Padovani, P., Giommi, P., \& Rau, A. 2012, MNRAS, 422, L48

Poole, T. S., Breeveld, A. A., Page, M. J., et al. 2008, MNRAS, 383, 627

Predehl, P., \& Schmitt, J. H. M. M. 1995, A\&A, 293, 889

Rau, A., Schady, P., Greiner, J., et al. 2012, A\&A, 538, A26

Rector, T. A., Stocke, J. T., Perlman, E. S., Morris, S. L., \& Gioia, I. M. 2000 AJ, 120, 1626

Romani, R. W. 2006, AJ, 132, 1959

Schlafly, E. F., \& Finkbeiner, D. P. 2011, ApJ, 737, 103

Shaw, M. S., Romani, R. W., Cotter, G., et al. 2013, ApJ, 764, 135

Skrutskie, M. F., Cutri, R. M., Stiening, R., et al. 2006, AJ, 131, 1163

Stecker, F. W., Malkan, M. A., \& Scully, S. T. 2012, ApJ, 761, 128

Tavecchio, F., Ghisellini, G., Ghirlanda, G., Foschini, L., \& Maraschi, L. 2010, MNRAS, 401, 1570

Urry, C. M., \& Padovani, P. 1995, PASP, 107, 803

Watson, D. 2011, A\&A, 533, A16

Zhang, J., Liang, E.-W., Zhang, S.-N., \& Bai, J. M. 2012, ApJ, 752, 157 